\title{
A novel Amh-Treck transgenic mouse line allows toxin-dependent loss of supporting cells in gonads
}

\author{
Mai Shinomura, Kasane Kishi, Ayako Tomita, Miyuri Kawasumi ${ }^{1}$, Hiromi Kanezashi, \\ Yoshiko Kuroda, Naoki Tsunekawa, Aisa Ozawa, Yoshimi Aiyama, Asuka Yoneda, Hitomi Suzuki ${ }^{1}$, \\ Michiko Saito ${ }^{2}$, Jean-Yves Picard ${ }^{3}$, Kenji Kohno ${ }^{2}$, Masamichi Kurohmaru, Masami Kanai-Azuma ${ }^{1}$ \\ and Yoshiakira Kanai
}

\begin{abstract}
Department of Veterinary Anatomy, The University of Tokyo, Yayoi 1-1-1, Bunkyo-ku, Tokyo 113-8657, Japan, ${ }^{1}$ Department of Experimental Animal Model for Human Disease, Center for Experimental Animals, Tokyo Medical and Dental University, Yushima, Bunkyo-ku, Tokyo 113-8510, Japan, ${ }^{2}$ Graduate School of Biological Sciences, Nara Institute of Science and Technology, 8916-5, Takayama, Ikoma, Nara 630-0192, Japan and ${ }^{3}$ INSERM U1133, BFA, University Paris VII, 75205 Paris Cedex 13, France
\end{abstract}

Correspondence should be addressed to Y Kanai; Email: aykanai@mail.ecc.u-tokyo.ac.jp

\begin{abstract}
Cell ablation technology is useful for studying specific cell lineages in a developing organ in vivo. Herein, we established a novel antiMüllerian hormone $(A M H)$-toxin receptor-mediated cell knockout (Treck) mouse line, in which the diphtheria toxin (DT) receptor was specifically activated in Sertoli and granulosa cells in postnatal testes and ovaries respectively. In the postnatal testes of Amh-Treck transgenic (Tg) male mice, DT injection induced a specific loss of the Sertoli cells in a dose-dependent manner, as well as the specific degeneration of granulosa cells in the primary and secondary follicles caused by DT injection in $\mathrm{Tg}$ females. In the testes with depletion of Sertoli cell, germ cells appeared to survive for only several days after DT treatment and rapidly underwent cell degeneration, which led to the accumulation of a large amount of cell debris within the seminiferous tubules by day 10 after DT treatment. Transplantation of exogenous healthy Sertoli cells following DT treatment rescued the germ cell loss in the transplantation sites of the seminiferous epithelia, leading to a partial recovery of the spermatogenesis. These results provide not only in vivo evidence of the crucial role of Sertoli cells in the maintenance of germ cells, but also show that the Amh-Treck Tg line is a useful in vivo model of the function of the supporting cell lineage in developing mammalian gonads.

Reproduction (2014) $148 \mathrm{H} 1-\mathrm{H} 9$
\end{abstract}

\section{Introduction}

In mammalian embryos at early organogenic stages, undifferentiated genital ridges start to develop into the testis or ovary dependent on their genetic sex. As the first morphological sign of testis differentiation, germ cells are surrounded by differentiating Sertoli cells, leading to the formation of testis cords (future seminiferous tubules), a tubular structure of the germ and Sertoli cells packed within the basal lamina sheath (Harikae et al. 2013a, Svingen \& Koopman 2013). In developing ovaries, both germ and pre-granulosa cells also aggregate to form the poorly defined ovarian cords (ovigerous cord), subsequently resulting in the formation of ovarian cysts, a closed sack-like structure of several oocytes and their surrounding pre-granulosa cells (Pepling 2006, Hummitzsch et al. 2013). After birth, the testis cords develop into the seminiferous tubules associated with active spermatogenesis, while in the ovary the ovarian cyst breaks down into each individual primordial follicle, an oocyte surrounded by a single layer of granulosa cells. A part of the primordial follicle continuously grows into primary and subsequent secondary follicle stages at the medullary side during the initial round of folliculogenesis (Mork et al. 2012, Harikae et al. 2013b).

From the fetal to adult stages, both Sertoli and granulosa cells secrete various growth factors to support the proliferation, differentiation and maintenance of the germ cells (Matsui 1998, Choi \& Rajkovic 2006, Buratini \& Price 2011, Zhang et al. 2011, Feng et al. 2014). Moreover, the germ cells physically contact and interact intimately with the supporting cells inside the basal lamina sheath. These histological and physiological findings indicate the crucial roles of the gonadal supporting cells in the maintenance and survival of germ cells in mammalian gonads. However, the duration 
for which the germ cells are maintained after the specific loss of supporting cells in vivo remains unclear.

A conditional in vivo depletion of lineage-specific cells can be achieved through administration of diphtheria toxin (DT) to toxin-receptor-mediated cellknockout (Treck)-transgenic (Tg) mice that carry a human DT receptor (DTR; HBEGF-mut) cDNA transgene driven by a cell linage-specific promoter (Saito et al. 2001). In this Treck system, the administration of DT to the Treck Tg mice causes depletion in only DTR-expressing cell linages and specifically eliminates these target cells in vivo (Takada et al. 2008, Matsuoka et al. 2013).

In mice, anti-Müllerian hormone $(A m h)$ is expressed specifically in the gonadal-supporting cell lineages: Sertoli and granulosa cells of postnatal immature gonads in both males and females (Lécureuil et al. 2002). Herein, we established three Amh-Treck Tg lines carrying the Amh-promoter-fused DTR cassette, in which conditional in vivo deletion of the gonadalsupporting cells was induced by DT injection at postnatal timepoints. Here we show a great potential in the Amh-Treck line as an in vivo functional assay system of the supporting cell lineage in mammalian gonads.

\section{Materials and methods}

\section{Amh-Treck Tg mouse line}

The Amh-Treck transgene was prepared by inserting the entire $5^{\prime}$-flanking promoter region of the Amh gene (Guerrier et al. 1990) upstream of DT receptor (DTR; I117V/L148V mutant of human HB-EGF tagged with EGFP; Furukawa et al. 2006). We successfully established three Amh-Treck Tg lines (\#36, \#85, \#94) with testis-specific expression of DTR in male pups (Supplementary Figure S1, see section on supplementary data given at the end of this article). The C57BL/6-Tg (CAG-EGFP) mice (GFP mice; SLC) and C57BL/6-R26-H2B-mCherry knockin mice (Cherry mice; Abe et al. 2011) were used as transplant donors for the rescue experiment. The ICR and C57BL/6 male mice (E12.5-10 weeks old; SLC) were also used for the immunohistochemical analyses. The animal experiments were carried out in accordance with the Guidelines for Animal Use and Experimentation of the University of Tokyo (approval IDs: P12-660 and P12-764).

\section{Quantitative RT-PCR}

Total RNA was reverse-transcribed using random primer with a Superscript-III cDNA Synthesis Kit (Invitrogen). PCR was carried out using an Applied Biosystems Step-One Real-Time PCR System with the primers and probes of Egfp (Mr04329676_mr) and Gapdh (Taqman control reagents).

\section{DT treatment}

Amh-Treck Tg mice (P3-21) were treated with DT (SigmaAldrich, $0.04-40.0 \mu \mathrm{g} / \mathrm{kg}$, i.p.) as described previously (Saito et al. 2001, Takada et al. 2008, Matsuoka et al. 2013).
All tissues were subjected to histological and immunohistochemical analyses as described below.

\section{Transplantation of EGFP or Cherry-positive Sertoli cells}

A single-cell suspension (including gonocytes and Sertoli cells) $\left(1.0 \times 10^{8} \mathrm{cell} \mathrm{s} / \mathrm{ml}\right)$ was prepared from the testes of 1 -week-old GFP/Cherry mice (C57BL6) by a two-step enzymatic digestion procedure (Shinohara et al. 2003), and then transplanted into the testes of 3-week-old recipient Tg males pre-treated with DT (0.4$4.0 \mu \mathrm{g} / \mathrm{kg}$ ). On days 10 and 45 after transplantation, all recipient Tg testes were removed from the tunica albuginea and roughly dissected in cold PBS under an Olympus fluorescence microscope (BX51N-34-FL2) and stereomicroscope (SZX16 plus U-LH100HG) (Nagai et al. 2012). The tissues were processed using the immunohistochemical techniques described below.

\section{Histology and immunohistochemistry}

The tissues were fixed in 4\% paraformaldehyde (PFA) or Bouin's solution and routinely embedded in paraffin. The sections ( $4 \mu \mathrm{m}$ thickness) were subjected to conventional histological (hematoxylin and eosin; H\&E) and immunohistochemical staining as described previously (Hiramatsu et al. 2010, Harikae et al. 2013b).

In brief, for immunohistochemical staining, the sections were incubated with anti-AMH (1:100 dilution; sc-6886; Santa Cruz), anti-3ß-HSD (1:2000 dilution; sc-30821; Santa Cruz), antiCherry (1:200 dilution; ab125096; Abcam, Cambridge, United Kingdom), anti-E-cadherin (1:500 dilution; 610181; BD-Pharmingen, Franklin Lakes, NJ, USA), anti-FOXL2 (1:200 dilution; Uda et al. (2004); 1:200 dilution, ab5096; Abcam), anti-GATA4 (1:250 dilution; sc-1237; Santa Cruz), anti-GFP (1:200 dilution; 598; MBL, Nagoya, Japan), anti-GFR $\alpha 1$ (1:100 dilution; AF560; R\&D Systems, Minneapolis, MN, USA), anti-HuB/Elavl2 (1:1000 dilution; H1538; Sigma), anti-laminin (1:400 dilution; 10765; ICN Pharmaceuticals, Santa Ana, CA, USA), anti-MVH/ DDX4 (1:5000 dilution; Toyooka et al. (2000); 1:1000 dilution; ab13840; Abcam), anti-PLZF (1:500 dilution; OP128, Calbiochem, Darmstadt, Germany), anti- $\alpha$-smooth muscle actin (antiSMA; 1:5000 dilution, A2547; Sigma), or anti-SOX9 (1:250 dilution; Kidokoro et al. (2005)) antibody at $4{ }^{\circ} \mathrm{C}$ for $12 \mathrm{~h}$. The reaction was visualized using a biotin-conjugated secondary antibody in combination with the Elite ABC Kit (Vector Laboratories) or by secondary antibodies conjugated with Alexa-488/594. TUNEL assays were also performed by using the Apoptotic Detection Kit (MK500; TaKaRa, Shiga, Japan).

\section{Results and discussion}

\section{Establishment of Amh-Treck Tg mouse lines}

The transgene was constructed as the Amh promoterdriven DT receptor (DTR; HBEGF-mut) (I117V/L148V mutant of human HB-EGF) tagged with EGFP (Furukawa et al. 2006; Fig. 1A). We obtained three Tg lines (healthy and fertile in both sexes) by pronuclear injection of the plasmid-based transgene into the C57BL6 background. In Wt mice, endogenous Amh expression is found in the 


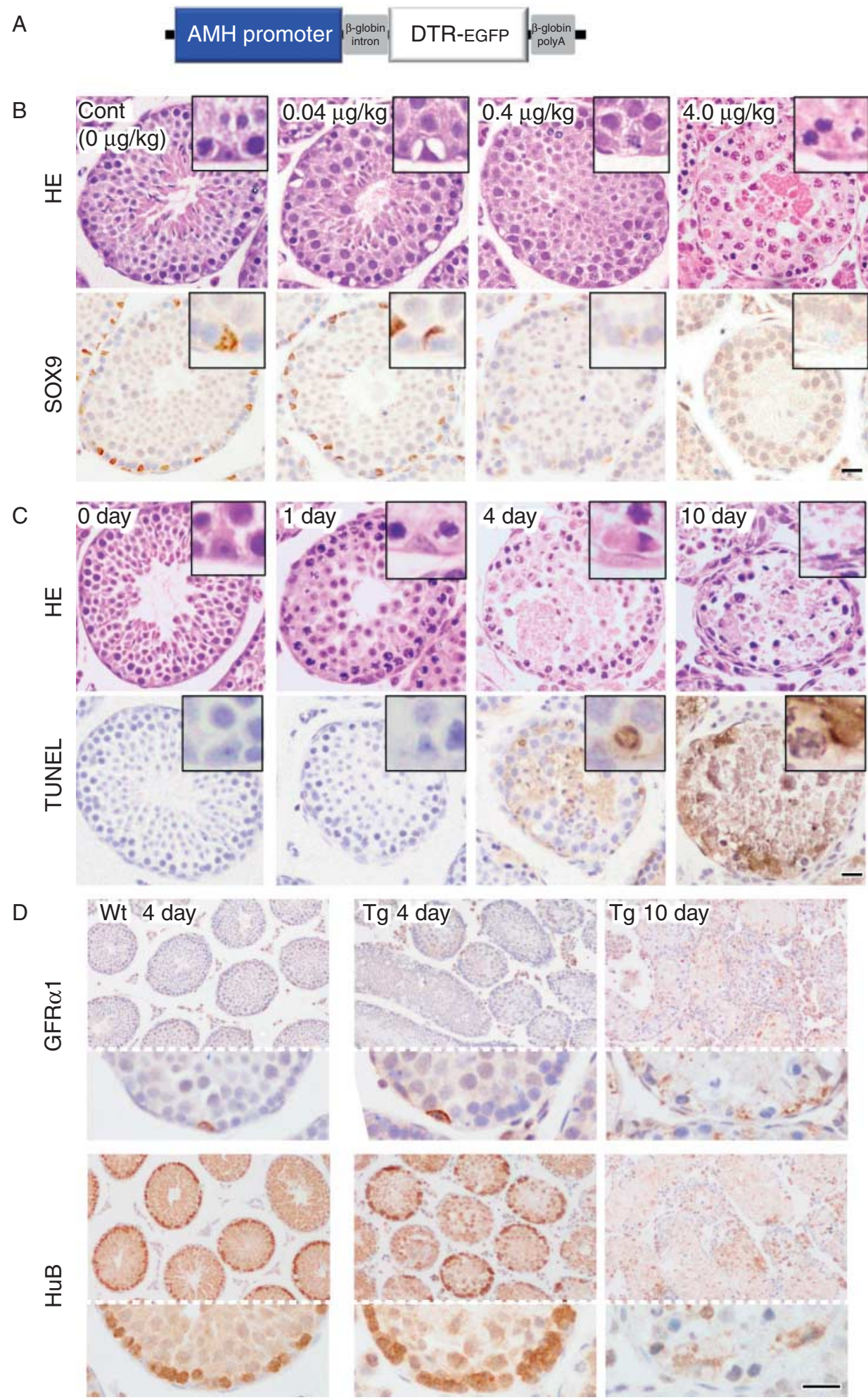

Figure 1 Characterization of $A M H$-Treck $T g$ lines (\#94). (A) Schematic representation showing the transgene of $A M H$ promoter-driven diphtheria toxin receptor (DTR; HBEGF-mut) fused with EGFP. (B) H\&E staining and anti-SOX9 immunostaining of the testes on day 4 after DT treatment, showing a dose-dependent degeneration of SOX9-positive Sertoli cells in Tg males treated with DT at 0, 0.04, 0.4, and 4.0 $\mu \mathrm{g} / \mathrm{kg}$. (C) H\&E and TUNEL staining showing a time-course pattern of the Sertoli cell and germ cell degeneration in the Tg males treated with DT at $4.0 \mu \mathrm{g} / \mathrm{kg}$. (D) AntiGFR $\alpha 1$ and HuB immunostaining of the testes on day 4 (two left-handed rows) and day 10 (most right-handed row) after DT treatment, showing the dynamics of undifferentiated $(\mathrm{GFR} \alpha 1+$ ) and differentiated $(\mathrm{HuB}+)$ spermatogonia in the Wt and Tg littermates treated with DT at $4.0 \mu \mathrm{g} / \mathrm{kg}$. Both GFR $\alpha 1+$ and $\mathrm{HuB}+$ populations of the spermatogonia disappear by day 10 after DT treatment. Scale bars represent $20 \mu \mathrm{m}$. 
Sertoli cells of the testes from embryonic day (E) 12.5 to postnatal day $(\mathrm{P}) 14$ (Supplementary Figure S2, see section on supplementary data given at the end of this article) and then almost repressed by P24 (Al-Attar et al. 1997). In the ovary, Amh expression is defined in the growing follicles after P3 and later stages (Lécureuil et al. 2002). The preliminary experiments carried out with DT treatment $(4.0 \mu \mathrm{g} / \mathrm{kg}$; Matsuoka et al. 2013) in the postnatal testes at P3-P10 showed degeneration in the majority of Sertoli cells in all of the three lines as well as no appreciable defects in other organs (Fig. 1 and Supplementary Figure S3). Among these three lines, the EGFPtagged DTR expression was, although fluorescent signals are very weak, detectable in the seminiferous tubules and the primordial follicles of the testes and ovaries in the $\# 94$ line (Supplementary Figure S4), in addition to the gonadspecific high-level expression of the DTR transgene by qPCR (Supplementary Figure S1). Moreover, even a lowdose DT treatment $(0.04 \mu \mathrm{g} / \mathrm{kg})$ can affect the nuclear shape of Sertoli cells and their arrangement in the \#94 line (see Fig. 1B), and thus we used mainly the $\# 94$ line for subsequent experiments.

\section{DT injection induces Sertoli cell degeneration in postnatal testes of $\mathrm{Tg}$ males in a dose-dependent manner}

First, we examined the dose-dependent effects of DT on the Sertoli cell degeneration in the Amh-Treck line. The $\mathrm{Tg}$ males and their Wt littermates at P21 (post-weaning) were treated with DT at various dosages, from 0.04 to $40.0 \mu \mathrm{g} / \mathrm{kg}$, and their organs including testes were histopathologically examined on day 4 after DT treatment (Fig. 1B and Supplementary Figure S5, see section on supplementary data given at the end of this article). In the $\mathrm{Tg}$ males treated with $0.04 \mu \mathrm{g} / \mathrm{kg}$ DT, most of Sox9-positive Sertoli cells appeared morphologically healthy but the nucleus position was arranged irregularly in the seminiferous epithelia as compared with the nontreated control testes (Fig. 1B). DT treatment at either 0.4 or $4.0 \mu \mathrm{g} / \mathrm{kg}$ clearly induced the loss of the Sox9-positive Sertoli cells in the seminiferous tubules by day 4 after DT treatment (Fig. 1B). In contrast, no appreciable defects were found in the Wt mice treated with DT at $4.0-40 \mu \mathrm{g} / \mathrm{kg}$ (Supplementary Figure S5). In addition, no gross-anatomical and histological defects were observed in the other organs of both Wt and $\mathrm{Tg}$ males treated with $4.0-40 \mu \mathrm{g} / \mathrm{kg}$ DT (Supplementary Figure S5; data not shown).

Next, we examined the temporal changes in the testicular phenotype in DT-treated Tg testes $(4.0 \mu \mathrm{g} / \mathrm{kg})$ between days 1 and 10 after DT treatment (Fig. 1C). On day 1 , DT treatment showed onset of Sertoli cell degeneration; however, histological loss of the nucleus was observed on day 4 after DT treatment. Moreover, histological analysis revealed that the germ cells appeared to undergo germ cell degeneration with visible accumulation of cell debris at the luminal side on day 4 .
Finally, by day 10 after DT treatment, all Sertoli and germ cells were degenerated in the seminiferous tubules and consequently, a large amount of cell debris had accumulated inside the seminiferous tubules (Fig. 1C). TUNEL staining also confirmed the appearance of apoptotic cell death in the germ cells at luminal side by day 4 after DT treatment (lower plates in Fig. 1C; Supplementary Figure S6, see section on supplementary data given at the end of this article). Anti-GFR $\alpha 1$ and anti-HuB immunostaining revealed that the GFR $\alpha 1+$ undifferentiated spermatogonia (including spermatogenic stem cells; Sato et al. 2011) and HuB + differentiated spermatogonia (including pre-leptotene spermatocytes; Supplementary Figure S7) are detectable in the basal compartment of the seminiferous tubules on day 4 after DT treatment. However, both spermatogonial populations were depleted by day 10 after DT treatment (Fig. 1D). These findings, therefore, confirmed that loss of Sertoli cells led to rapid cell death of germ cells within several days. This is consistent with previous reports involving Sertoli cell-specific deletion of Wt1 (Gao et al. 2006) or Sox9/Sox8 (Barrionuevo et al. 2009) gene(s), which demonstrated a drastic reduction in germ cell number in the postnatal testes, together with defective Sertoli cell function.

\section{Sertoli cell-specific loss of the seminiferous tubules in DT-treated Tg testes at P3 and P21}

The specificity of the cell depletion in DT-treated testes was examined on day 4 after DT treatment. The male Tg pups and their Wt littermates at P3 and P21 were treated with DT $(4.0 \mu \mathrm{g} / \mathrm{kg})$ and the expression patterns of various somatic cell markers were examined immunohistochemically (Fig. 2A). In P3 testes treated with DT (upper plates in Fig. 2B), the almost complete depletion of the Amh-positive or Sox9-positive Sertoli cells was found throughout the entire testis area. This is in sharp contrast with E-cad-positive rete testis epithelia, which appeared histologically normal (Fig. 2B). Immunohistochemical analyses of P21 testes on day 4 after DT treatment also confirmed that GATA4-positive cells appeared intact in the peritubular and interstitial regions. However, no GATA4-positive signals were detectable in the seminiferous epithelia, leading to $\mathrm{MVH}$-positive germ cells occupying the inside of the tubules (Fig. 2C). The laminin-positive basal lamina, SMA-positive pertibular myoid cells, and 33-HSDpositive Leydig cells appeared intact in the interstitial region (Fig. 2C). The complete degeneration of cells inside the tubules by day 10 after DT treatment indicates that Sertoli cell-specific depletion occurs by day 4; germ cells then undergo rapid degeneration, which may result in the accumulation of a large amount of cell debris at the luminal side by day 10 after DT treatment. 
A $\quad$ DT i.p.

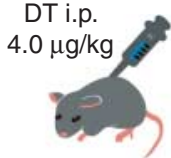

$\begin{array}{ll}\text { P3 } & \\ \text { P21 } 4 \text { day Immunohistochemistry }\end{array}$

B

E-CAD

$\mathrm{AMH}$

sox9

E-CAD
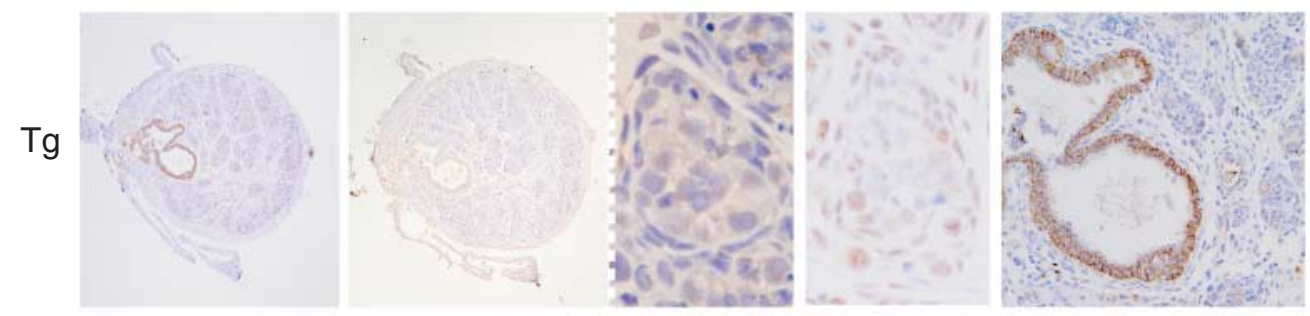

Wt
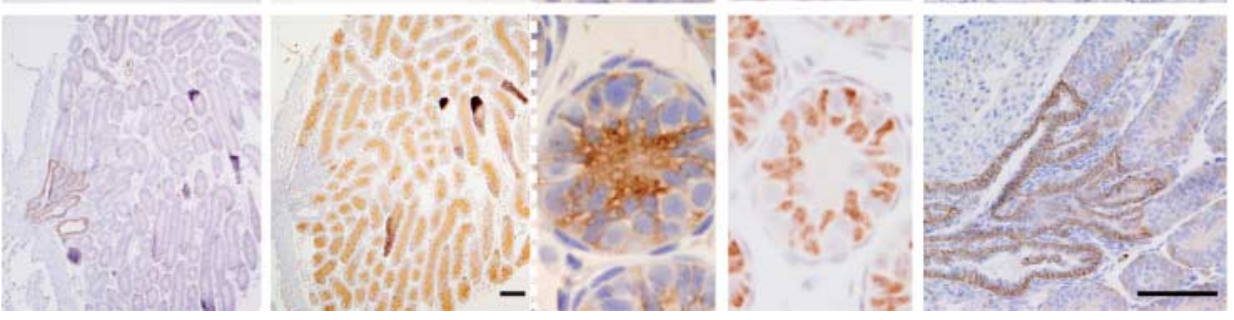

C

GATA4
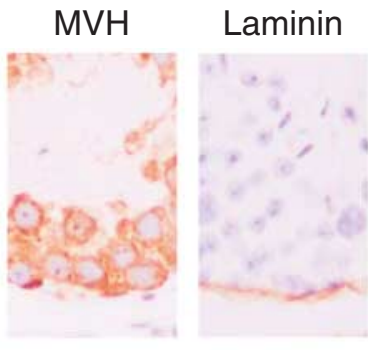

SMA

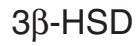

$\mathrm{Tg}$
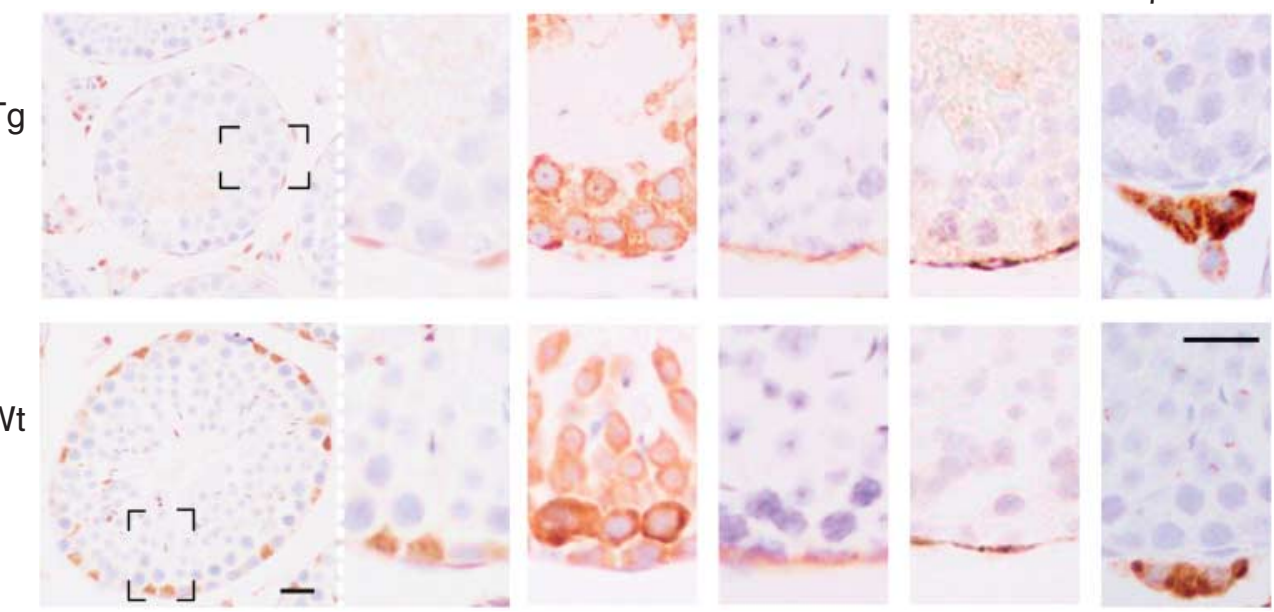

Figure $2 \mathrm{~A}$ specific loss of the Sertoli cells in postnatal testes of DT-treated Tg males. (A) Schematic representation of DT treatment. Both Tg and Wt littermates at P3 and P21 were treated with DT at $4.0 \mu \mathrm{g} / \mathrm{kg}$ and their testes were examined histopathologically on day 4 after DT treatment. (B) AntiE-cadherin (E-CAD), anti-AMH and anti-SOX9 immunostaining of Tg and Wt testes treated with DT at P3. The DT-treated testis shows a reduced size of the testicular parenchyma only in the Tg line, in which AMH-/SOX9-positive Sertoli cells are almost completely missing throughout the testicular parenchyma. In contrast, E-cad-positive rete testis epithelia are normally found in the same Tg testis (the plate farthest to the right in B). No histopathological defects in the DT-treated testis are detectable in the Wt littermate (lower plates in B). (C) Anti-GATA4, anti-MVH, anti-laminin, anti-SMA and anti-3 $\beta-H S D$ immunostaining of Tg and $\mathrm{Wt}$ testes treated with DT at P21. GATA4-positive nuclear signals are missing inside the seminiferous tubules. No appreciable differences in anti-laminin-/SMA-/3 $\beta$-HSD-staining patterns are detectable between Tg and Wt testes. Scale bars represent $100 \mu \mathrm{m}$ in $\mathrm{B} ; 20 \mu \mathrm{m}$ in $\mathrm{C}$.

\section{Transplantation of healthy immature Sertoli cells can rescue the germ cell degeneration in DT-treated Tg testes, leading to a partial recovery of the spermatogenesis}

The intratubular injection method of the cell suspension prepared from immature testes previously showed that immature dividing Sertoli cells selectively colonize and support the recipient-derived spermatogenesis in the empty seminiferous tubules pre-treated with the Buslfan and cadmium (Shinohara et al. 2003). In order to rescue the germ cell degeneration, healthy immature Sertoli cells (around P7) labeled by cytoplasmic/nuclear EGFP 
A

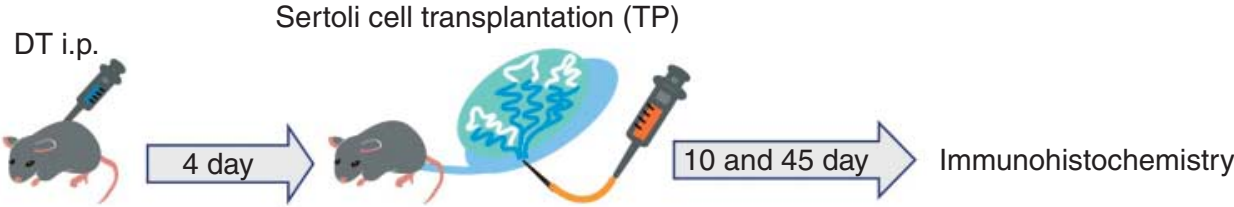

$\mathrm{B}$

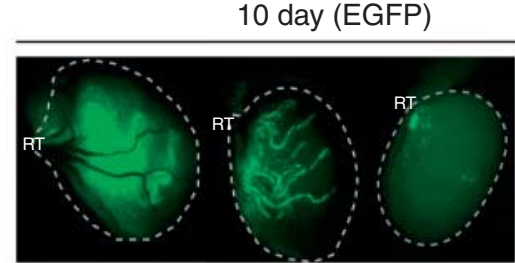

$\mathrm{DT}+\mathrm{TP}$

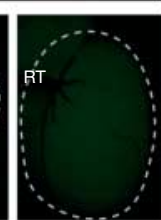

Cont

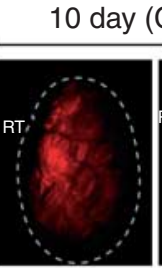

DT + TP

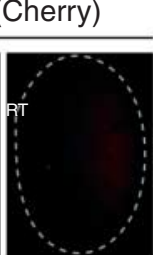

Cont
45 day (Cherry)

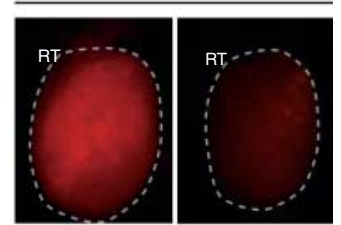

$\mathrm{DT}+\mathrm{TP}$

Cont
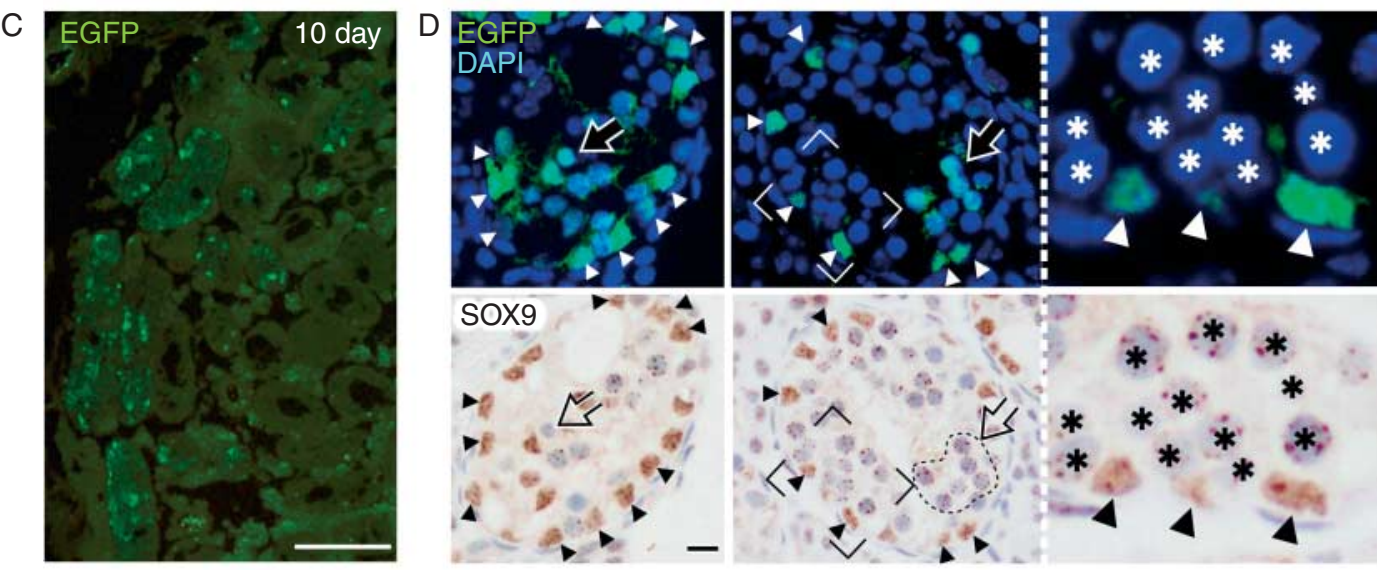

E
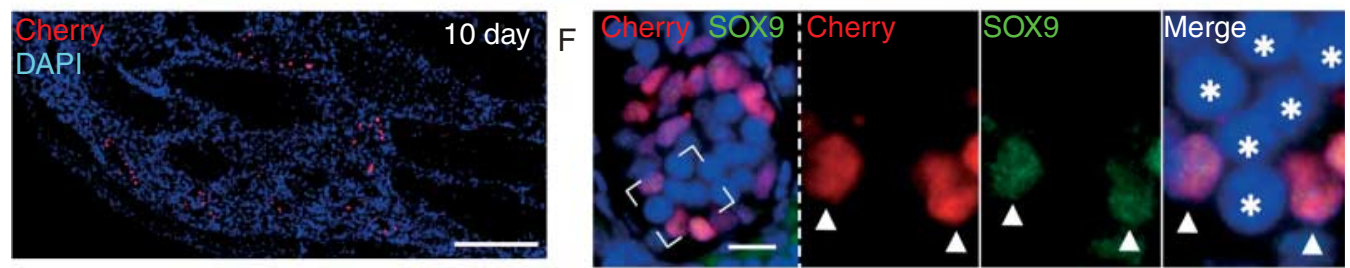

G
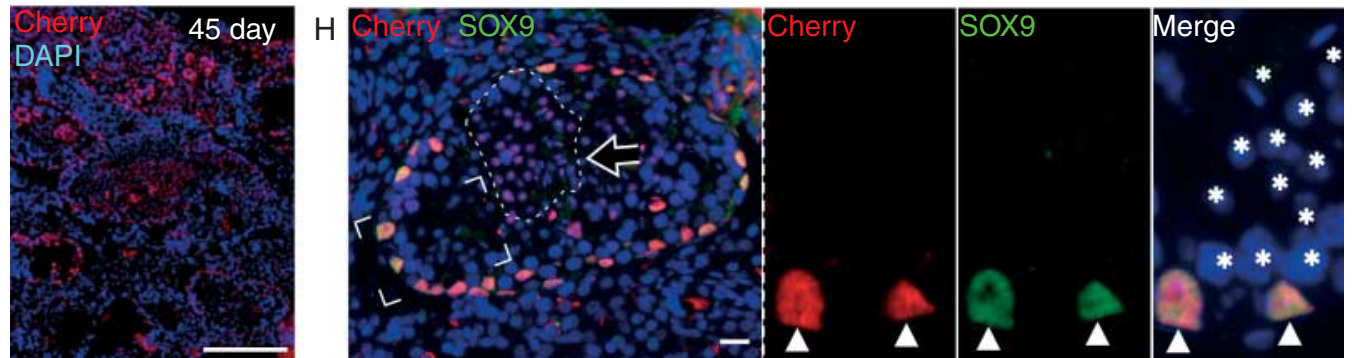

Figure $3 \mathrm{~A}$ rescue experiment by transplantation of the healthy Sertoli cells into DT-treated Tg testes. (A) Schematic representation of Sertoli cell transplantation on day 4 and day 45 after DT injection $(0.4-4.0 \mu \mathrm{g} / \mathrm{kg}$ ). (B) Various degrees of contribution of donor-derived (EGFP/Cherry-positive) Sertoli cells in each testis on day 10 and day 45 after Sertoli cell transplantation. (C, D, E, and F) Donor-derived cytoplasmic/nuclear EGFP and nuclear Cherry signals in the transplanted tubules (lower magnified images; C and E) and EGFP/Cherry signals (green/red) and anti-SOX9

(brown in D, green in F) immunostaining images of the same testicular section (D and F), showing the contribution of donor-derived healthy Sertoli cells (arrowheads) in the DT-treated testis on day 10 after transplantation. In the most right-handed images of plates D and F, transplanted Sertoli cells directly support the host-derived germ cells (asterisks) in DT-treated Tg testes. (G and H) Anti-Cherry (red) and anti-SOX9 (green in H) double immunostaining images showing spermatogenic patches derived from both endogenous (asterisks) and exogenous (open arrow) germ cells in the transplanted DT-treated Tg testis on Day 45 after transplantation. Transplanted Sertoli cells directly support spermatogenesis of the host-derived germ cells (asterisks). In the host, plates $\mathrm{C}$ and $\mathrm{D}$ are the testis pre-treated with DT at $0.4 \mu \mathrm{g} / \mathrm{kg}$, while plates $\mathrm{E}, \mathrm{F}, \mathrm{G}$, and $\mathrm{H}$ are the testes pre-treated with DT at $4.0 \mu \mathrm{g} / \mathrm{kg}$. Scale bars represent $200 \mu \mathrm{m}$ in C, E, and G; $10 \mu \mathrm{m}$ in D; $20 \mu \mathrm{m}$ in F, H. 
A

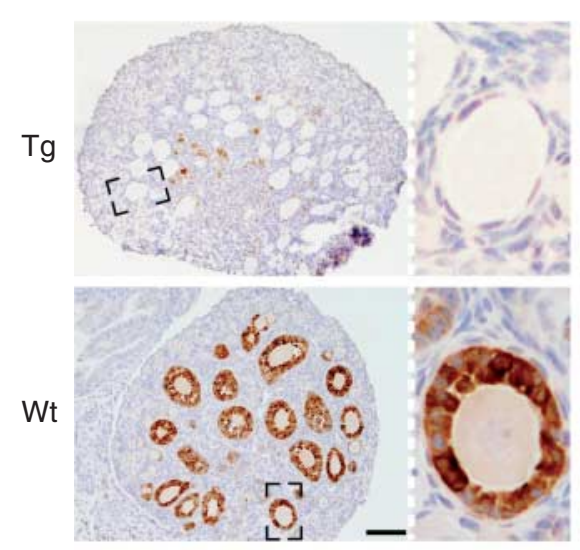

B

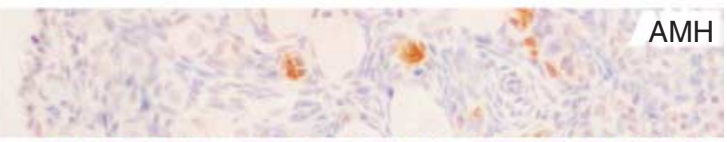

$\mathrm{Tg}$

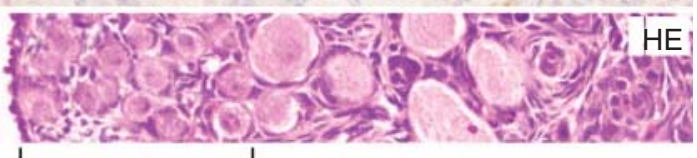

Wt

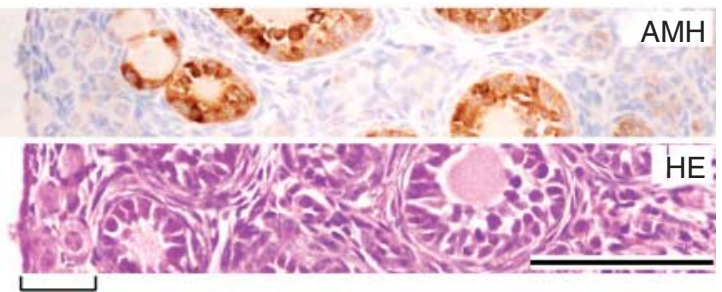

C

HE

FOXL2

MVH

Laminin

SMA

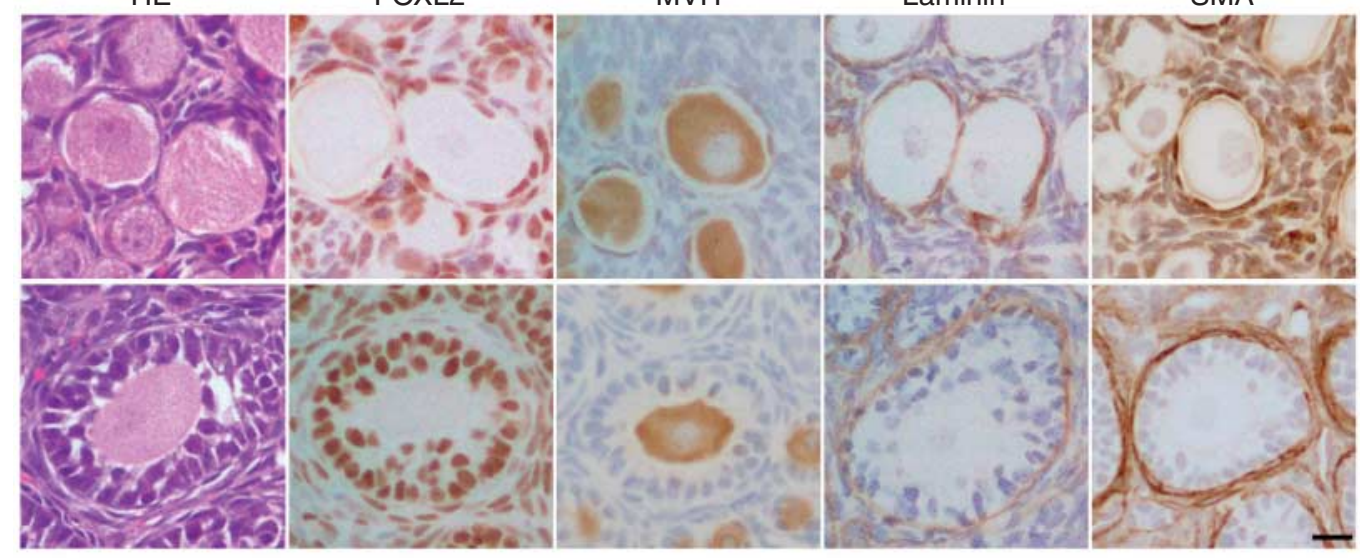

D

HE

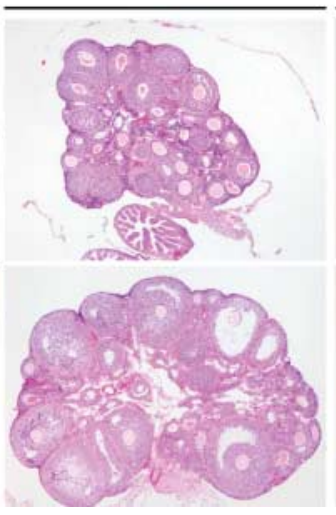

$3 \beta-H S D$

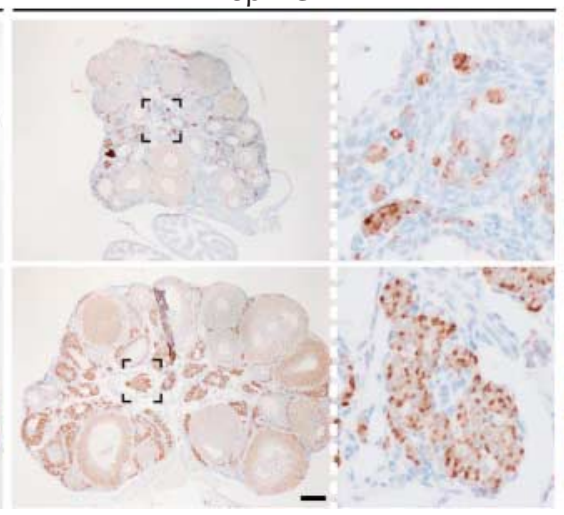

$\mathrm{AMH}$

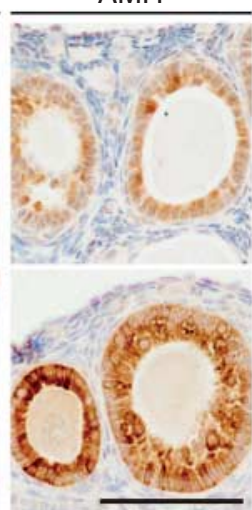

Figure 4 Specific depletion of the granulosa cells of AMH-positive presumptive primary and secondary follicles in developing ovaries. (A and B) Anti-AMH immunostaining and H\&E staining images of the P7 ovaries of Tg and Wt littermates (DT; $40.0 \mu \mathrm{g} / \mathrm{kg}$ treatment at P3). In B, the ovarian surface region including an $\mathrm{AMH}$-negative primordial follicle pool (indicated by bars) is shown on the left side. (C) Higher magnified images of $\mathrm{H} \& \mathrm{E}$, anti-FOXL2, anti-MVH, anti-laminin and anti-SMA staining patterns in the presumptive primary and secondary follicles located in the centromedullary region of DT-treated ovaries (Tg: upper plates, Wt: lower plates). FOXL2-positive granulosa cells are missing in the affected follicles of the DT-treated Tg ovary, despite no differences in anti-laminin- /anti-SMA-immunostaining patterns between Tg and Wt ovaries.

(D) Gross-anatomical, H\&E staining and anti-3ß-HSD/anti-AMH immunostaining images of the P28 ovaries of Tg and Wt littermates treated with DT $(40.0 \mu \mathrm{g} / \mathrm{kg})$ at P3 (day 25 after DT treatment). The new recruitment of AMH-positive primary and secondary follicles is shown in the DT-treated Tg ovary, and both the ovary size and the $3 \beta$-HSD-positive area are reduced in the Tg ovary. Nuclei are counterstained with hematoxylin in blue. In A and D, each inset shows a higher-magnification image of the area indicated by the broken rectangle. Scale bars represent $100 \mu \mathrm{m}$ in $A, B$, and D; $20 \mu \mathrm{m}$ in $\mathrm{C}$. 
(CAG-EGFP) or nuclear Cherry (R26-H2B-mCherry) were transplanted (i.e. intratubular injection) in P21 Tg males, on day 4 after DT treatment $(0.4-4.0 \mu \mathrm{g} / \mathrm{kg})$. The transplanted testes were examined on day 10 after Sertoli cell transplantation (Fig. 3A). In the DT-treated Tg testes transplanted with exogenous healthy testis cells, the transplantation sites were visualized by means of EGFPor Cherry-positive signals under a dissecting microscope (number of testes with donor cells per total testes injected: 10/18 testes (EGFP, 4/8 testes; Cherry, 6/10 testes); Fig. 3B). Histological analysis revealed that a majority of transplanted cells were positive for Sox9, a Sertoli cell marker, suggesting selective settlement of the exogenous healthy Sertoli cells inside the DT-treated seminiferous tubules (Fig. 3D). Moreover, exogenous healthy Sertoli cells were shown to maintain both endogenous and exogenous spermatogenic cells at the transplantation sites (Fig. 3C, D, E, and F). In some areas, the transplanted Sertoli cells had replaced the degenerated Sertoli cells and contributed to supporting the hostderived germ cells on day 10 (most right plates in Fig. 3D and F). Next, we examine the phenotypes of the DT-treated Tg testes on day 45 after the Sertoli cell transplantation. In one sample (Cherry, 1/3 testes), the transplanted Sertoli cells were observed to survive in the DT-treated testes (Fig. 3B, G, and Supplementary Figure S8, see section on supplementary data given at the end of this article). Moreover, in some areas, it was shown that the transplanted Sertoli cells partially restore spermatogenesis of endogenous spermatogenic cells on day 45 after the transplantation (Fig. $3 \mathrm{H}$ and Supplementary Figure S8). These findings show that the transplanted immature Sertoli cells could replace some degenerated Sertoli cells and rescue in part the germ cell degeneration, albeit locally, in DT-treated Tg testes. These data also suggest this $A M H$-Treck system is useful for transplantation studies in terms of evaluating Sertoli cell function. Indeed, this new Treck system is more simple and effective than the previous method of the Sertoli cell transplantation, which used an intratubular injection of cadmium to deplete Sertoli cells (Shinohara et al. 2003).

\section{DT treatment allows the loss of granulosa cells under high-dose DT treatment in postnatal ovaries}

Finally, we examined the phenotype of DT-induced cell depletion in the initial round of folliculogenesis in the $\mathrm{Tg}$ females at P7 (on day 4 after DT treatment at P3). Since the preliminary experiments using DT treatment at $4.0 \mu \mathrm{g} / \mathrm{kg}$ resulted in no appreciable histological defects in the developing ovaries (data not shown), we applied DT treatment $(40.0 \mu \mathrm{g} / \mathrm{kg})$ to the $\mathrm{Tg}$ ovaries; the phenotype was examined histopathologically on day 4 or day 25 after DT injection at P3 (Fig. 4). A high-dose DT treatment induced almost complete loss of the $\mathrm{AMH}$-positive follicles in the centromedullary region of the ovaries (Fig. 4A and B). At this DT concentration, both histological and immunohistochemical analyses confirmed the lack of granulosa cells around the growing oocyte in the majority of presumptive primary and secondary follicles (Fig. 4B and C). The FOXL2-positive presumptive theca cells were found in the ovarian interstitium around the growing follicles. Both laminin-positive basal lamina and SMApositive perifollicular cells appeared intact and were directly connected to the growing oocyte with a diameter similar to those of the primary and secondary follicle stages (Fig. 4C). No appreciable defects were detected in the ovaries of the littermates treated with DT at the same dose (' $\mathrm{Wt}^{\prime}$ ' in Fig. 4B and C). The absence of any histological abnormality in the ovarian-surface primordial follicle pool of the same DT-treated ovaries (Fig. 4B) indicated a specific loss of the granulosa cells in growing follicles. Moreover, this suggested that the sensitivity to DT treatment of the granulosa cells of female pups was lower than that of the Sertoli cells of male pups in this Amh-Treck line. On day 30 after DT treatment $(40 \mu \mathrm{g} / \mathrm{kg})$, the DT-treated ovaries displayed a recovery of folliculogenesis in the cortex region, although the ovary size and the $3 \beta-H S D$-positive interstitial region appeared to be reduced (Fig. 4D). The DT-treated ovaries contained many healthy Amh-positive primordial and secondary follicles (the plate farthest to the right in Fig. 4D), suggesting new recruitment of primary and secondary follicles in these DT-treated ovaries. This was also consistent with the absence of any appreciable defects in the primordial follicle pool after the high-dose DT treatment.

In conclusion, we have established a novel Treck system that permits the specific loss of gonadal supporting cells at the postnatal stages using DT treatment. The present data confirm the essential role of Sertoli cells in germ cell survival in vivo. This model system is also useful not only for in vivo analyses of structural and endocrine roles of the supporting cell lineage at the postnatal stages, but also potentially for the in vivo functional assay of defective supporting cells in stem cell niche and spermatogenesis.

\section{Supplementary data}

This is linked to the online version of the paper at http://dx.doi. org/10.1530/REP-14-0171.

\section{Declaration of interest}

The authors declare that there is no conflict of interest that could be perceived as prejudicing the impartiality of the research reported.

\section{Funding}

This work was supported by a grant from the Ministry of Education, Science, Sports and Culture of Japan to (S-24228005, PA- 23132504, PA-25132703, and C-24500485). M Shinomura and Y Aiyama are DC1 JSPS Research Fellows. 


\section{Author contribution statement}

M Shinomura and K Kishi contributed equally to this work as first authors. M Kanai-Azuma and Y Kanai contributed equally to this work for last authors.

\section{Acknowledgements}

The authors are grateful to Drs H Yonekawa, C Taya, and $K$ Matsuoka for their kind and useful advice for the Treck system, to Drs Y Furuta, T Fujimori, and T Abe for the gift of the R26-H2B-mCherry transgenic mice and to Ms Y Uchiyama and I Yagihashi for their technical and secretarial assistance.

\section{References}

Abe T, Kiyonari H, Shioi G, Inoue K, Nakao K, Aizawa S \& Fujimori T 2011 Establishment of conditional reporter mouse lines at ROSA26 locus for live cell imaging. Genesis 49 579-590. (doi:10.1002/dvg.20753)

Al-Attar L, Noël K, Dutertre M, Belville C, Forest MG, Burgoyne PS, Josso N \& Rey R 1997 Hormonal and cellular regulation of Sertoli cell anti-Müllerian hormone production in the postnatal mouse. Journal of Clinical Investigation 100 1335-1343. (doi:10.1172/JCI119653)

Barrionuevo F, Georg I, Scherthan H, Lecureuil C, Guillou F, Wegner M \& Scherer G 2009 Testis cord differentiation after the sex determination stage is independent of Sox 9 but fails in the combined absence of Sox 9 and Sox8. Developmental Biology 327 301-312. (doi:10.1016/j.ydbio. 2008.12.011)

Buratini J \& Price CA 2011 Follicular somatic cell factors and follicle development. Reproduction, Fertility, and Development 23 32-39. (doi:10.1071/RD10224)

Choi Y \& Rajkovic A 2006 Genetics of early mammalian folliculogenesis. Cellular and Molecular Life Sciences 63 579-590. (doi:10.1007/s00018005-5394-7)

Feng CW, Bowles J \& Koopman P 2014 Control of mammalian germ cell entry into meiosis. Molecular and Cellular Endocrinology 382 488-497. (doi:10.1016/j.mce.2013.09.026)

Furukawa N, Saito M, Hakoshima T \& Kohno K 2006 A diphtheria toxin receptor deficient in epidermal growth factor-like biological activity. Journal of Biochemistry 140 831-841. (doi:10.1093/jb/mvj216)

Gao F, Maiti S, Alam N, Zhang Z, Deng JM, Behringer RR, Lecureuil C, Guillou F \& Huff V 2006 The Wilms tumor gene, Wt1, is required for Sox9 expression and maintenance of tubular architecture in the developing testis. PNAS 103 11987-11992. (doi:10.1073/pnas. 0600994103)

Guerrier D, Boussin L, Mader S, Josso N, Kahn A \& Picard JY 1990 Expression of the gene for anti-Müllerian hormone. Journal of Reproduction and Fertility 88 695-706. (doi:10.1530/jrf.0.0880695)

Harikae K, Miura K \& Kanai Y 2013a Early gonadogenesis in mammals: significance of long and narrow gonadal structure. Developmental Dynamics 242 330-338. (doi:10.1002/dvdy.23872)

Harikae K, Miura K, Shinomura M, Matoba S, Hiramatsu R, Tsunekawa N, Kanai-Azuma M, Kurohmaru M, Morohashi K \& Kanai Y 2013b Heterogeneity in sexual bipotentiality and plasticity of granulosa cells in developing mouse ovaries. Journal of Cell Science 126 2834-2844. (doi:10.1242/jcs.122663)

Hiramatsu R, Harikae K, Tsunekawa N, Kurohmaru M, Matsuo I \& Kanai Y 2010 FGF signaling directs a center-to-pole expansion of tubulogenesis in mouse testis differentiation. Development 137 303-312. (doi:10.1242/ dev.040519)

Hummitzsch K, Irving-Rodgers HF, Hatzirodos N, Bonner W, Sabatier L, Reinhardt DP, Sado Y, Ninomiya Y, Wilhelm D \& Rodgers RJ 2013 A new model of development of the mammalian ovary and follicles. PLOS ONE 8 e55578. (doi:10.1371/journal.pone.0055578)

Kidokoro T, Matoba S, Hiramatsu R, Fujisawa M, Kanai-Azuma M, Taya C, Kurohmaru M, Kawakami H, Hayashi Y, Kanai Y et al. 2005 Influence on spatiotemporal patterns of a male-specific Sox9 activation by ectopic Sry expression during early phases of testis differentiation in mice. Developmental Biology 278 511-525. (doi:10.1016/j.ydbio.2004.11. 006)

Lécureuil C, Fontaine I, Crepieux P \& Guillou F 2002 Sertoli and granulosa cell-specific Cre recombinase activity in transgenic mice. Genesis 33 114-118. (doi:10.1002/gene.10100)

Matsui Y 1998 Regulation of germ cell death in mammalian gonads. APMIS: Acta Pathologica, Microbiologica, et Immunologica Scandinavica 106 142-147; discussion 147-148. (doi:10.1111/j.1699-0463. 1998.tb01329.x)

Matsuoka K, Saito M, Shibata K, Sekine M, Shitara H, Taya C, Zhang X, Takahashi TA, Kohno K, Kikkawa Y et al. 2013 Generation of mouse models for type 1 diabetes by selective depletion of pancreatic $\beta$ cells using toxin receptor-mediated cell knockout. Biochemical and Biophysical Research Communications 436 400-405. (doi:10.1016/ j.bbrc.2013.05.114)

Mork L, Maatouk DM, McMahon JA, Guo JJ, Zhang P, McMahon AP \& Capel B 2012 Temporal differences in granulosa cell specification in the ovary reflect distinct follicle fates in mice. Biology of Reproduction $\mathbf{8 6}$ 1-9. (doi:10.1095/biolreprod.111.095208)

Nagai R, Shinomura M, Kishi K, Aiyama Y, Harikae K, Sato T, KanaiAzuma M, Kurohmaru M, Tsunekawa N \& Kanai Y 2012 Dynamics of GFR $\alpha 1$-positive spermatogonia at the early stages of colonization in the recipient testes of W/Wv male mice. Developmental Dynamics 241 1374-1384. (doi:10.1002/dvdy.23824)

Pepling ME 2006 From primordial germ cell to primordial follicle: mammalian female germ cell development. Genesis 44 622-632. (doi:10.1002/dvg.20258)

Saito M, Iwawaki T, Taya C, Yonekawa H, Noda M, Inui Y, Mekada E, Kimata Y, Tsuru A \& Kohno K 2001 Diphtheria toxin receptor-mediated conditional and targeted cell ablation in transgenic mice. Nature Biotechnology 19 746-750. (doi:10.1038/90795)

Sato T, Aiyama Y, Ishii-Inagaki M, Hara K, Tsunekawa N, Harikae K, Uemura-Kamata M, Shinomura M, Zhu XB, Maeda S et al. 2011 Cyclical and patch-like GDNF distribution along the basal surface of Sertoli cells in mouse and hamster testes. PLOS ONE 6 e28367. (doi:10.1371/journal. pone.0028367)

Shinohara T, Orwig KE, Avarbock MR \& Brinster RL 2003 Restoration of spermatogenesis in infertile mice by Sertoli cell transplantation. Biology of Reproduction 68 1064-1071. (doi:10.1095/biolreprod.102. 009977)

Svingen T \& Koopman P 2013 Building the mammalian testis: origins, differentiation, and assembly of the component cell populations. Genes and Development 27 2409-2426. (doi:10.1101/gad.228080.113)

Takada T, Shitara H, Matsuoka K, Kojima E, Ishii R, Kikkawa Y, Taya C, Karasuyama H, Kohno K \& Yonekawa H 2008 A novel hairless mouse model on an atopic dermatitis-prone genetic background generated by receptor-mediated transgenesis. Transgenic Research 17 1155-1162. (doi:10.1007/s11248-008-9203-6)

Toyooka Y, Tsunekawa N, Takahashi Y, Matsui Y, Satoh M \& Noce T 2000 Expression and intracellular localization of mouse Vasa-homologue protein during germ cell development. Mechanisms of Development 93 139-149. (doi:10.1016/S0925-4773(00)00283-5)

Uda M, Ottolenghi C, Crisponi L, Garcia JE, Deiana M, Kimber W, Forabosco A, Cao A, Schlessinger D \& Pilia G 2004 Foxl2 disruption causes mouse ovarian failure by pervasive blockage of follicle development. Human Molecular Genetics 13 1171-1181. (doi:10. 1093/hmg/ddh124)

Zhang L, Tang J, Haines CJ, Feng HL, Lai L, Teng X \& Han Y 2011 c-kit and its related genes in spermatogonial differentiation. Spermatogenesis $\mathbf{1}$ 186-194. (doi:10.4161/spmg.1.3.17760)

Received 31 March 2014

First decision 22 April 2014

Revised manuscript received 14 August 2014

Accepted 8 September 2014 